\title{
A Relation Between the Coefficients and Roots of Two Equations and Its Application to Diophantine Problems*
}

\author{
T. N. Sinha**
}

(October 3, 1969)

The object of this paper is to prove:

THEOREM 1: The system of equations

$$
a_{1}^{r}+a_{2}^{r}+\ldots+a_{n}^{r}=b_{1}^{r}+b_{2}^{r}+\ldots+b_{n}^{r}(r=1,2, \ldots, j-1, j+1, \ldots, n+1)
$$

where $\mathrm{j}$ is odd, has no nontrivial solutions in positive integers.

THEOREM 2: No two equations $\mathrm{a}_{1}^{\mathrm{r}}+\mathrm{a}_{2}^{\mathrm{r}}=\mathrm{b}_{1}^{\mathrm{r}}+\mathrm{b}_{2}^{\mathrm{r}}(\mathrm{r}=\mathrm{s}, \mathrm{t})$ where the pairs of values of $\mathrm{r}$ range between 1 and 4 can have a nontrivial solution in integers.

Key words: Diophantine equations; Prouhet-Tarry-Escott problem; symmetric functions.

In section 1 of this paper we indicate a general relation between the coefficients of two polynomial equations of the same degree $n \geqslant 1$ and the differences of the sums of like powers of the roots of the equations. In section 2 we apply this relation and deduce some results concerning the problem of equal sums of like powers in number theory.

1. Let $\left\{a_{1}, a_{2}, \ldots, a_{n}\right\}$ and $\left\{b_{1}, b_{2}, \ldots ., b_{n}\right\}$ be two sets of numbers, not all necessarily rational in any set. Let the elements $a_{i}$ of the first set be the roots of the equation

$$
x^{n}-p_{1} x^{n-1}+\ldots+(-1)^{n} p_{n}=0
$$

and so also let the elements $b_{i}$ of the second set be the roots of the equation

$$
y^{n}-q_{1} y^{n-1}+\ldots+(-1)^{n} q_{n}=0 .
$$

In what follows we write

$$
A_{r}=a_{1}^{r}+a_{2}^{r}+\ldots \ldots+a_{n}^{r}, B_{r}=b_{1}^{r}+b_{2}^{r}+\ldots .+b_{n}^{r}
$$

where $r$ is an integer $\geqslant 1$. By Newton's formula on the sum of the powers of the roots of an equation we have from equation (1),

$$
A_{r}-p_{1} A_{r-1}+p_{2} A_{r-2}-\ldots+(-1)^{r-1} p_{r-1} A_{1}+(-1)^{r} r p_{r}=0
$$

for $r=1,2,3, \ldots$. with $p_{r}=0$ if $r>n$. Similarly, from equation (2)

$$
B_{r}-q_{1} B_{r-1}+q_{2} B_{r-2}-\ldots+(-1)^{r-1} q_{r-1} B_{1}+(-1)^{r} r q_{r}=0
$$

\footnotetext{
*An invited paper.

** Present address: Department of Mathematics, Bhagalpur University, Bhagalpur-7, Bihar, India.
} 
for $r=1,2,3, \ldots$. with $q_{r}=0$ if $r>n$. Eliminating $p_{1}, p_{2}, \ldots p_{r-1}$ between the first $r$ equations in (4), we easily obtain

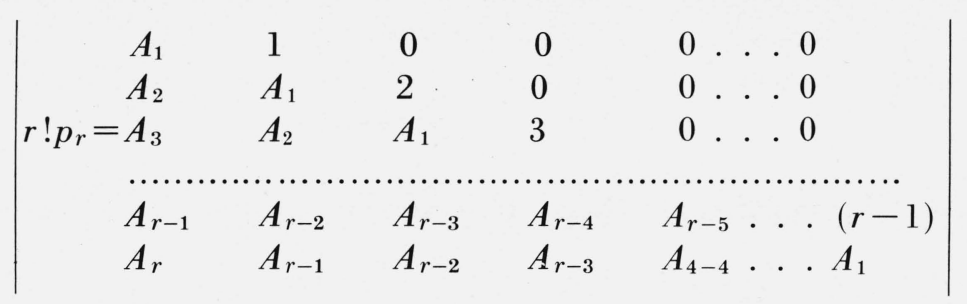

with a similar expression for $r ! q_{r}$. Let us write

$$
A_{r}=B_{r}+h_{r} \quad(r=1,2,3, \ldots)
$$

and let $H_{r}$ denote the determinant obtained by replacing $A_{j}$ by $h_{j}$ in the determinant in (6). When the $A_{j}$ are so replaced, $r ! p_{r}$ is replaced by $H_{r}$ and by symmetry we have, corresponding to the relation (4),

$$
h_{r}-\frac{H_{1}}{1 !} h_{r-1}+\frac{H_{2}}{2 !} h_{r-2}-\ldots \ldots+(-1)^{r} r \frac{H_{r}}{r !}=0 .
$$

Writing $c_{r}=r ! p_{r}$ and $d_{r}=r ! q_{r}$ we then get

$$
\begin{aligned}
c_{1} & =d_{1}+H_{1}, H_{1}=h_{1} \\
c_{2} & =A_{1}^{2}-A_{2}=\left(B_{1}+h_{1}\right)^{2}-\left(B_{2}+h_{2}\right)=\left(B_{1}^{2}-B_{2}\right)+2 h_{1} B_{1}+h_{1}^{2}-h_{2} \\
& =d_{2}+2 H_{1} d_{1}+H_{2}=d_{2}+\left(\begin{array}{l}
2 \\
1
\end{array}\right) H_{1} d_{1}+\left(\begin{array}{l}
2 \\
2
\end{array}\right) H_{2} \\
c_{3} & =A_{1}^{3}-3 A_{1} A_{2}+2 A_{3}=\left(B_{1}+h_{1}\right)^{3}-3\left(B_{1}+h_{1}\right)\left(B_{2}+h_{2}\right)+2\left(B_{3}+h_{3}\right) \\
& =d_{3}+3 H_{1} d_{2}+3 H_{2} d_{1}+H_{3}=d_{3}+\left(\begin{array}{l}
3 \\
1
\end{array}\right) H_{1} d_{2}+\left(\begin{array}{l}
3 \\
2
\end{array}\right) H_{2} d_{1}+\left(\begin{array}{l}
3 \\
3
\end{array}\right) H_{3} .
\end{aligned}
$$

Let us assume that

$$
c_{m}=d_{m}+\left(\begin{array}{c}
m \\
1
\end{array}\right) H_{1} d_{m-1}+\left(\begin{array}{c}
m \\
2
\end{array}\right) H_{2} d_{m-2}+\ldots+\left(\begin{array}{c}
m \\
m
\end{array}\right) H_{m}
$$

for $m=1,2, \ldots .,(r-1), r \geqslant 2$. Then (4) gives by virtue of (7),

$$
\begin{array}{r}
\left(B_{r}+h_{r}\right)-\frac{1}{1 !}\left(d_{1}+H_{1}\right)\left(B_{r-1}+h_{r-1}\right)+\frac{1}{2 !}\left[d_{2}+\left(\begin{array}{l}
2 \\
1
\end{array}\right) H_{1} d_{1}+\left(\begin{array}{l}
2 \\
2
\end{array}\right) H_{2}\right]\left(B_{r-2}+h_{r-2}\right)-\ldots \\
\ldots+(-1)^{r-1} \frac{1}{(r-1) !}\left[d_{r-1}+\left(\begin{array}{c}
r-1 \\
1
\end{array}\right) H_{1} d_{r-2}+\ldots+\left(\begin{array}{c}
r-1 \\
r-1
\end{array}\right) H_{r-1}\right]\left(B_{1}+h_{1}\right) \\
+(-1)^{r} \frac{r c_{r}}{r !}=0, r \geqslant 2 .
\end{array}
$$

If we denote the left-hand side of (5) and (8) by $Q_{r}$ and $T_{r}$ respectively, then arranging the terms in (9) we get

$$
\begin{aligned}
& {\left[Q_{r}-(-1)^{r} \frac{r d_{r}}{r !}\right]-\frac{H_{1}}{1 !}\left[Q_{r-1}-(-1)^{r-1} \frac{(r-1) d_{r-1}}{(r-1) !}\right]+\ldots} \\
& +(-1)^{r-1} \frac{H_{r-1}}{(r-1) !}\left[Q_{1}-(-1)^{1} \frac{1 d_{1}}{1 !}\right]+\left[T_{r}-(-1)^{r} \frac{r H_{r}}{r !}\right]-\frac{d_{1}}{1 !}\left[T_{r-1}-(-1)^{r-1} \frac{(r-1) H_{r-1}}{(r-1) !}\right] \\
& +\ldots+(-1)^{r-1} \frac{d_{r-1}}{(r-1) !}\left[T_{1}-(-1)^{1} \frac{1 H_{1}}{1 !}\right]+(-1)^{r} \frac{r c_{r}}{r !}=0
\end{aligned}
$$


Noting that $Q_{j}=T_{j}=0, j=1,2,3, \ldots$, the above easily gives

$-\frac{r d_{r}}{r !}-\frac{H_{1}}{(r-1) ! 1 !}(r-1+1) d_{r-1}-\frac{H_{2}}{(r-2) ! 2 !}(r-2+2) d_{r-2}-\ldots$.

that is,

$$
-\frac{H_{r-1}}{1 !(r-1) !} d_{1}-\frac{r H_{r}}{r !}+\frac{r c_{r}}{r !}=0
$$

$$
c_{r}=d_{r}+\left(\begin{array}{l}
r \\
1
\end{array}\right) H_{1} d_{r-1}+\left(\begin{array}{l}
r \\
2
\end{array}\right) H_{2} d_{r-2}+\ldots . .+\left(\begin{array}{l}
r \\
r
\end{array}\right) H_{r}
$$

Hence, by induction, it follows that (10) holds for all $r=1,2,3, \ldots$., (where, of course, $c_{r}=d_{r}=0$ if $r>n)$.

2. We now restrict the elements $a_{i}$ and $b_{i}$ of the preceding section to be all integers. The notations continue to remain the same as before and in what follows now all letters denote integers. If we put in (7) $h_{r}=0$ for $r=s, t, \ldots$., there results a system of equations with equal sums of like powers of the type

$$
A_{r}=B_{r}(r=s, t, \ldots)
$$

where $A_{r}$ and $B_{r}$ are given by (3). Conversely, a system (11) makes in (7) $h_{r}=0, r=s, t, \ldots$. Using the relation (10) of the previous section we shall now deduce that certain systems (11) do not possess nontrivial solutions in integers.

I. If the $a$ 's and $b$ 's satisfy (11) with $r=1,2, \ldots, n$, then since $h_{j}=0(j=1,2, \ldots . n)$, the relation (10) at once yields the well known Bastien's theorem [1] $]^{1}$

II. Let the $a_{i}$ and $b_{i}$ be positive integers which satisfy the system (11) for $r=1,2, \ldots, j-1$, $j+1, \ldots ., n+1$. Then $c_{r}$ and $d_{r}$ are all positive for $r=1,2, \ldots, n$ and we have $h_{1}=h_{2}$ $=\ldots .=h_{j-1}=h_{j+1} \ldots \ldots=h_{n+1}=0$. Without loss of generality we may suppose $h_{j} \geqslant 0$. Then by (8)

$$
H_{r}=(-1)^{j-1} \frac{(r-1) !}{(r-j) !} H_{r-j} h_{j}
$$

From the relation (12), it follows that $H_{r}=0$ for $r=1,2, \ldots,(j-1)$ and for $r=j$

$$
H_{j}=(-1)^{j-1}(j-1) ! h_{j}
$$

If $r>j$, the same relation shows that (since $H_{r}$ is expressed in terms of $H_{r-j}$ and $H_{r}=0$ for $r=1,2, \ldots(j-1)) H_{r}=0$ unless $r$ is a multiple of $j$. However, if $r$ is a multiple of $j$, say $r=j k$, we have by repeated application of (12)

$$
\begin{aligned}
H_{j k} & =(-1)^{(j-1)(k-1)} \frac{(j k-1) !(j k-j-1) ! \ldots(2 j-1) !}{(j k-j) !(j k-2 j) ! \ldots j !} H_{j} h_{j}^{k-1} \\
& =(-1)^{(j-1) k} \frac{(j k-1) !(j k-j-1) ! \ldots(2 j-1) !}{(j k-j) !(j k-2 j) ! \cdots j !}(j-1) ! h_{j}^{k},(13)
\end{aligned}
$$

Now if $j$ is odd, we have by (14), $H_{j k} \geqslant 0$, the sign of equality occurring only when $h_{j}=0$. Assuming that $j$ is odd, if we substitute the values of $H_{r}(r=1,2, \ldots, n+1)$, (which is either zero or positive as obtained in (14)), in the relation (10) with $r=n+1$, then, since $c_{n+1}=d_{n+1}=0$, the L.H.S. is zero while the R.H.S. would be positive unless $h_{j}=0$. In the latter case, however, the solutions are only trivial by Bastien's theorem. We have thus proved

THEOREM 1: The system of equations

$$
a_{1}^{r}+a_{2}^{r}+\ldots+a_{n}^{r}=b_{1}^{r}+b_{2}^{r}+\ldots+b_{n}^{r}(r=1,2, \ldots, j-1, j+1, \ldots, n+1),
$$

where $\mathrm{j}$ is odd, has no nontrivial solutions in positive integers.

\footnotetext{
${ }^{1}$ Figures in brackets indicate the literature references at the end of this paper.
} 
This confirms an assertion of Gupta (sec. 3, [3]) made there without proof. The case $j=1$ gives precisely his theorem (sec. 1 [3]).

III. To treat the pair of equations

$$
a_{1}^{r}+a_{2}^{r}=b_{1}^{r}+b_{2}^{r} \quad(r=s, t)
$$

we may apply the relations of the previous section with $n=2$. Without loss of generality one can suppose that $s$ and $t$ are relatively prime in (15). For sake of simplicity we consider here only such pairs of values of $r$ in (15) as range between 1 and 4. We then need consider the system (15) only with the following pairs of values of $r$ :

$$
r=1,2 ; \quad 1,3 ; \quad 1,4 ; \quad 3,4 ; \quad 2,3 .
$$

(a) $r=1,2$. In this case, the system (15) has only trivial solutions by Bastien's theorem.

(b) $r=1,3$. In this case, the system (15) implies the system

$$
\left(w+a_{1}\right)^{r}+\left(w+a_{2}\right)^{r}+\left(w-b_{1}\right)^{r}+\left(w-b_{2}\right)^{r}=\left(w+b_{1}\right)^{r}+\left(w+b_{2}\right)^{r}+\left(w-a_{1}\right)^{r}+\left(w-a_{2}\right)^{r}
$$

for $r=1,2,3$ and 4, where $w$ is arbitrary. The latter system has only trivial solutions by Bastien's theorem and so has the system (15) in this case.

In each of the three remaining cases we actually calculate the coefficients of the quadratic equations (1) and (2) in terms of the $h$ 's by means of the relation (10) and then apply the condition for integral roots: $c_{1}^{2}-2 c_{2}=\square, \quad d_{1}^{2}-2 d_{2}=$

(c) $r=1,4$. In this case $h_{1}=h_{4}=0$ and (10) gives

$$
\begin{gathered}
c_{1}=d_{1}, \quad c_{2}=d_{2}-h_{2} \\
0=-3 h_{2} d_{1}+2 h_{3}, \quad 0=-6 h_{2} d_{2}+8 h_{3} d_{1}+3 h_{2}^{2}
\end{gathered}
$$

We may suppose $h_{2} \neq 0$, for otherwise (15) has only trivial solutions. From (16) we then obtain

$$
c_{1}=d_{1}=2 h_{3} / 3 h_{2}, c_{2}=\left(16 h_{3}^{2}-9 h_{2}^{3}\right) / 18 h_{2}^{2}, d_{2}=\left(16 h_{3}^{2}+9 h_{2}^{3}\right) / 18 h_{2}^{2} .
$$

Applying the condition for integral roots, we have

$$
-12 h_{3}^{2}+9 h_{2}^{3}=u_{1}^{2}, \quad-12 h_{3}^{2}-9 h_{2}^{3}=u_{2}^{2} .
$$

By addition, we see that (17) can hold only with $h_{3}=0$, in which case (15) has only trivial solutions by case (b). The same conclusion was arrived at by Gloden [2], using a different method.

(d) $r=3,4$. In this case $h_{3}=h_{4}=0$ and (10) gives

$$
\begin{gathered}
c_{1}=d_{1}+h_{1}, \quad c_{2}=d_{2}+2 h_{1} d_{1}+h_{1}^{2}-h_{2}, \quad 0=3 h_{1} d_{2}+3\left(h_{1}^{2}-h_{2}\right) d_{1}+h_{1}^{3}-3 h_{1} h_{2} \\
0=6\left(h_{1}^{2}-h_{2}\right) d_{2}+4\left(h_{1}^{3}-3 h_{1} h_{2}\right) d_{1}+h_{1}^{4}-6 h_{1}^{2} h_{2}+3 h_{2}^{2} .
\end{gathered}
$$

We may suppose that $h_{1} \neq 0$, for otherwise (15) has only trivial solutions by case (b). From (18) we then obtain

$$
\begin{aligned}
& c_{1}=h_{1}\left(h_{1}^{4}+2 h_{1}^{2} h_{2}+3 h_{2}^{2}\right) / 2\left(h_{1}^{4}+3 h_{2}^{2}\right), \\
& d_{1}=h_{1}\left(-h_{1}^{4}+2 h_{1}^{2} h_{2}-3 h_{2}^{2}\right) / 2\left(h_{1}^{4}+3 h_{2}^{2}\right) \\
& c_{2}=\left(h_{1}^{6}+3 h_{1}^{4} h_{2}+9 h_{1}^{2} h_{2}^{2}-9 h_{2}^{3}\right) / 6\left(h_{1}^{4}+3 h_{2}^{2}\right), \\
& d_{2}=\left(h_{1}^{6}-3 h_{1}^{4} h_{2}+9 h_{1}^{2} h_{2}^{2}+9 h_{2}^{3}\right) / 6\left(h_{1}^{4}+3 h_{2}^{2}\right) .
\end{aligned}
$$


Applying the condition for integral roots, we have

$$
3 h_{1}^{2}\left(h_{1}^{4} \pm 2 h_{1}^{2} h_{2}+3 h_{2}^{2}\right)^{2}-4\left(h_{1}^{4}+3 h_{2}^{2}\right)\left(h_{1}^{6} \pm 3 h_{1}^{4} h_{2}+9 h_{1}^{2} h_{2}^{2} \mp 9 h_{2}^{3}\right)=12 v_{1}^{2}, 12 v_{2}^{2} .
$$

Adding these two equations and simplifying, we get

$$
-h_{1}^{2}\left(h_{1}^{4}+9 h_{2}^{2}\right)^{2}=6\left(v_{1}^{2}+v_{2}^{2}\right)
$$

which can hold only with $h_{1}=0$, contrary to assumption. It follows that (15) has only trivial solutions in this case.

(e) $r=2,3$. In this case $h_{2}=h_{3}=0$ and (10) gives

$c_{1}=d_{1}+h_{1}, \quad c_{2}=d_{2}+2 h_{1} d_{1}+h_{1}^{2}, \quad 0=3 h_{1} d_{2}+3 h_{1}^{2} d_{1}+h_{1}^{3}=6 h_{1}^{2} d_{2}+4 h_{1}^{3} d_{1}+h_{1}^{4}-6 h_{4}$.

We may assume $h_{1} \neq 0$, for otherwise we have only trivial solutions. Then (19) gives

$$
c_{1}=\left(h_{1}^{4}-6 h_{4}\right) / 2 h_{1}^{3}, \quad d_{1}=-\left(h_{1}^{4}+6 h_{4}\right) / 2 h_{1}^{3}, \quad c_{2}, d_{2}=\left(h_{1}^{4} \mp 18 h_{4}\right) / 6 h_{1}^{2} .
$$

Applying the condition for integral roots

$$
-h_{1}^{8} \pm 36 h_{1}^{4} h_{4}+108 h_{4}^{2}=12 w_{1}^{2}, 12 w_{2}^{2} .
$$

Dividing by 3 and putting $6 h_{4}=f, h_{1}^{4}=g$ where $h_{1}$ and hence $g$ is divisible by $3,2 w_{1}=u, 2 w_{2}=v$ we have from (21)

$$
u^{4}+u^{2} v^{2}+v^{4}=3 z^{2}, \quad \text { where } z=f^{2}-g^{2} / 3 .
$$

The equation (22) is known to be impossible in integers except when $u^{2}=v^{2}=z$ (see for instance [5]). In the latter case, however, $w_{1}^{2}=w_{2}^{2}$ and by substraction (2l) gives $h_{1} h_{4}=0$. Thus, as $h_{1} \neq 0$ by assumption, $h_{4}=0$, in which case (15) has only trivial solutions by case (d). The same result was obtained by Schmidt [4] as a byproduct in discussing the rational points on curves of genus 1 . The preceding conclusions are summarized in:

THEOREM 2: No two equations (15) where the pairs of values of $\mathrm{r}$ range between 1 and 4 can have a nontrivial solution in integers.

Addendum: Theorem 1 is also true when $j$ is even $(1<j \leqslant n+1)$.

Proof. Let $j$ be even $(1<j \leqslant n+1)$. We may assume $h_{j}>0$ (the case $h_{j}=0$ gives only trivial solutions). If we set $n+1=q j+R, 0 \leqslant R<j$, then on putting $r=n+1$ in (10) we have (since $H_{r}=0$ unless $r=k j$ )

$$
0=\sum_{k=1}^{q}\left(\begin{array}{c}
n+1 \\
k j
\end{array}\right) H_{k j} d_{n+1-k j} \quad \text { with } \quad d_{n+1-q j}=d_{0}=1 \quad \text { if } \quad n+1=q j .
$$

If $q=1$, this equation is impossible, since the $d$ 's are positive and with $j$ even. $H_{j}<0$. Hence we take $q>1$. Substituting for $H_{k j}(k=1,2, \ldots, q)$ from (14) and dividing by $(j-1) ! h_{j}$ we get from the above equation

$$
\begin{aligned}
\left(\begin{array}{c}
n+1 \\
j
\end{array}\right) d_{n+1-j} & =\sum_{k=2}^{q}(-1)^{k}\left(\begin{array}{c}
n+1 \\
k j
\end{array}\right) \frac{(k j-1) !(k j-j-1) ! \ldots(2 j-1) !}{(k j-j) !(k j-2 j) ! \ldots j !} h_{j}^{k-1} d_{n+1-k j} \\
& =\sum_{k=2}^{q}(-1)^{k} \frac{(n+1) !}{(n+1-k j) !} \cdot \frac{h_{j}^{k-1} d_{n+1-k j}}{k ! j^{k-1} \cdot j !}
\end{aligned}
$$

whence

$$
d_{n+1-j}=(n+1-j) ! \sum_{k=2}^{q}(-1)^{k} \frac{h_{j}^{k-1} d_{n+1-k j}}{(n+1-k j) ! k ! j^{k-1}}
$$


Again from (10) with $r=n+1-j$ we have

$$
c_{n+1-j}=d_{n+1-j}+\sum_{k=2}^{q}\left(\begin{array}{c}
n+1-j \\
\overline{k-1} j
\end{array}\right) H_{\overline{k-1} j} d_{n+1-k j} .
$$

Substituting for $H_{\overline{k-1} j}$ and simplifying as above we have, since the $c$ 's are positive,

$$
d_{n+1-j}>\sum_{k=2}^{q}(-1)^{k} \frac{(n+1-j) !}{(n+1-k j) !} \cdot \frac{h_{j}^{k-1} d_{n+1-k j}}{(k-1) ! j^{k-1}}
$$

Hence from $\left(14_{1}\right)$ and $\left(14_{2}\right)$ we have

$$
\sum_{k=2}^{q}(-1)^{k} \frac{h_{j}^{k-1} d_{n+1-k j}}{(n+1-k j) ! k ! j^{k-1}}>\sum_{k=2}^{q}(-1)^{k} \frac{h_{j}^{k-1} d_{n+1-k j}}{(n+1-k j) !(k-1) ! j^{k-1}} .
$$

Transposing and removing the factor $h_{j} / j$ we have from above

whence

$$
\sum_{k=2}^{q}(-1)^{k-1} \frac{(k-1) h_{j}^{k-2} d_{n+1-k j}}{(n+1-k j) ! k ! j^{k-2}}>0
$$

$$
-d_{n+1-2 j}+2 !(n+1-2 j) ! \sum_{k=3}^{q}(-1)^{k-1} \frac{(k-1) h_{j}^{k-2} d_{n+1-k j}}{(n+1-k j) ! k ! j^{k-2}}>0
$$

Again from (10) with $r=n+1-2 j$ we have

$$
c_{n+1-2 j}=d_{n+1-2 j}+\sum_{k=3}^{q}\left(\begin{array}{c}
n+1-2 j \\
\overline{k-2} j
\end{array}\right) H_{\overline{k-2 j}} d_{n+1-k j}
$$

and therefore as above

$$
d_{n+1-2 j}+(n+1-2 j) ! \sum_{k=3}^{q}(-1)^{k-2} \frac{k(k-1) h_{j}^{k-2} d_{n+1-k j}}{(n+1-k j) ! k ! j^{k-2}}>0 .
$$

Adding $\left(14_{4}\right)$ and $\left(14_{5}\right)$ and removing the factor $(n+1-2 j) ! h_{j} / j$ we get

$$
\sum_{k=3}^{q}(-1)^{k-2} \frac{(k-1)(k-2) h_{j}^{k-3} d_{n+1-k j}}{(n+1-k j) ! k ! j^{k-3}}>0 .
$$

Repeating the process, which reduces $\left(14_{3}\right)$ to $\left(14_{6}\right)$, with $\left(14_{6}\right)$ and so in succession with each such resulting inequality we ultimately get

$$
(-1)^{q-(q-1)} \cdot \frac{(q-1)(q-2) \ldots(q-\overline{q-1}) h_{j}^{q-q} d_{n+1-q j}}{(n+1-q j) ! q ! j^{q-q}}>0
$$

i.e., $-d_{n+1-q j}>0$, which is clearly impossible since $d_{n+1-q j} \geqslant 1$.

I thank Professor H. Gupta who kindly gave some useful suggestions in the preparation of this paper.

\section{References}

[1] Bastien, L., Sphnix-Oedipe 8, 171-172 (1913).

[2] Gloden, A., Note on a system of Diophantine equations, Scripta Mathemetica 15, 163 (1949).

[3] Gupta, H., A system of equations having no nontrivial solutions, J. Res. Nat. Bur. Stand. (U.S.), 71 B (Math. and Math. Phys.) No. 4, 181-182 (1967).

[4] Schmidt, H., Uber einige diophantische, Aufgaben 3 und 4 grades, Jber Deutsch. Math. Verein 67, Abt 1, 2-13 (1964/65).

[5] Upensky, J. V., and Heaslet, M. A., Elementary Number Theory, chapt. XII, Ex. 9, 428 (McGraw Hill Book Co., New York, N.Y., 1939). 